\title{
Esqueletos inhumados en cementerios excavados en roca: el caso de Tejuela/Villanueva de Soportilla (Burgos)*
}

\author{
Skeletons buried in rock-cut cemeteries: the example of \\ Tejuela/Villanueva de Soportilla (Burgos)
}

\author{
José F. Martín-Alonso \\ Alumno de doctorado de la Universidad de Granada \\ josefcomartin@correo.ugr.es \\ ORCID iD: https://orcid.org/0000-0002-0540-9986 \\ Rosa M. Maroto Benavides \\ Laboratorio de Antropología. Universidad de Granada \\ rmmaroto@ugr.es \\ ORCID iD: https://orcid.org/0000-0003-4710-4977 \\ Sylvia A. Jiménez-Brobeil \\ Laboratorio de Antropología. Universidad de Granada \\ jbrobeil@ugr.es \\ ORCID iD: https://orcid.org/0000-0001-8758-5635
}

Enviado: 23-07-2019. Aceptado: 31-01-2020. Publicado online: 23-03-2021

Cómo citar este artículo / Citation: Martín-Alonso, J. F., Maroto Benavides, R. M. y Jiménez-Brobeil, S. A. (2021). "Esqueletos inhumados en cementerios excavados en roca: el caso de Tejuela/Villanueva de Soportilla (Burgos)". Archivo Español de Arqueología, 94, e03. DOI: https://doi.org/10.3989/aespa.094.021.03

RESUMEN: Se hace una aportación innovadora al estudio de los cementerios en roca desde una perspectiva antropológica. Se analizan 109 tumbas, que conservan restos óseos, del cementerio de Tejuela/Villanueva de Soportilla que fue excavado primeramente por Alberto del Castillo y después por Aratikos Arqueólogos. No hay una relación clara entre tipos de tumbas y sexo, aunque las tumbas de bañera son más comunes entre mujeres. No hay relación con la edad a excepción de los niños muy pequeños, que están enterrados mayoritariamente en tumbas del mismo tipo. Aunque la longitud de las tumbas está correlacionada con la estatura de los individuos, no puede establecerse con precisión la edad o sexo del sujeto que se inhumó en una sepultura que se encuentre vacía. Se incluyen dataciones absolutas obtenidas a partir de los esqueletos que aportan fechas entre el último tercio del siglo VIII y comienzos del XI.

\footnotetext{
* Este trabajo se ha realizado dentro del proyecto de investigación "Salud y alimentación en poblaciones rurales de la España medieval" (HAR2016-75788-P), financiado por el Ministerio de Economía y Competitividad del Gobierno de España (MINECO), dirigido desde la Universidad de Granada por S. A. Jiménez-Brobeil.
} 
Palabras clave: tipología de tumbas; datación absoluta; sexo; edad; tamaño.

ABSTRACT: This paper provides an innovative contribution to the study of rock cemeteries from an anthropological perspective. One hundred and nine tombs, which conserve bone remains, are analyzed from the Tejuela/ Villanueva de Soportilla cemetery which was first excavated by Alberto del Castillo and later on by Aratikos Arqueólogos. There is no clear relationship between types of tombs and sex, although bathtub tombs are more common among women. There is no relationship with age except for very young children, who are mostly buried in tombs of the same type. Although the length of the tombs is correlated with the stature of the individuals, the age or sex of the subject who is buried in an empty tomb cannot be established with precision. Absolute dating obtained from the skeletons are included, providing dates between the last third of the eighth century and the beginning of the eleventh century.

Keywords: burial typology; absolute chronology; sex; age; size.

Copyright: (C) 2021 CSIC. Este es un artículo de acceso abierto distribuido bajo los términos de la licencia de uso y distribución Creative Commons Reconocimiento 4.0 Internacional (CC BY 4.0).

\section{INTRODUCCIÓN}

Entre los cementerios de las comunidades altomedievales de parte del norte de la península ibérica los arqueólogos han prestado especial atención a los que presentan tumbas excavadas en la roca. La investigación científica de este tipo de sepulturas se inició en la década de los 60 del pasado siglo por parte del profesor Alberto del Castillo (1970 y 1972), considerado como uno de los fundadores de la Arqueología medieval española. Este investigador seguía las teorías del historiador Claudio Sánchez-Albornoz (1966) y vinculó la fundación y utilización de varios de estos cementerios con un movimiento de repoblación asociado a la Reconquista. En sus trabajos estableció una cronología basada en la tipología de las tumbas, elemento que también aplicó a la identificación de la posible edad o sexo de las personas inhumadas en ellas. Los estudios realizados desde entonces han demostrado numerosos errores en estos planteamientos y, por otra parte, han analizado estos cementerios desde diferentes puntos de vista como centros de poder o delimitación de territorios (Bolós y Pagés, 1982; Andrío, 1987; Härke, 2001; Quirós, 2006 y 2011; Martín Viso, 2007 y 2012; Padilla y Álvaro, 2010 y 2013; Tente 2015; López, Álvaro y Travé, 2016; Rubio, 2020). La bibliografía desarrollada es amplísima y convierte a estas tumbas excavadas en roca en uno de los paradigmas de la Arqueología altomedieval. Son muchas las cuestiones desarrolladas en su estudio y varias de las incógnitas planteadas derivan del hecho de que, en una amplia mayoría de casos, estas sepulturas se encontraron vacías y ello supuso un importante hándicap, por ejemplo, para su datación (Martín Viso, 2014).

El trabajo que aquí se expone está desarrollado sobre una de las pocas colecciones de restos humanos procedentes de un cementerio altomedieval que posee tumbas excavadas en roca. Se presenta, por tanto, más desde la perspectiva de la Antropología Física y por ello con una óptica que puede resultar un tanto diferente a la de los medievalistas. No pretende, en ningún caso, proporcionar nuevas interpretaciones de estos cementerios sino tan solo realizar una aportación a su conocimiento desde una perspectiva basada en la interdisciplinariedad de la Antropología y la Arqueología.

En 1970 el profesor Alberto del Castillo inició las excavaciones en uno de estos cementerios, el de Santa María de Tejuela, situado cerca de Villanueva de Soportilla en el término municipal de Bozoó (Burgos). Aunque en la literatura y desde su publicación en 1972, se conoce con el nombre de Villanueva de Soportilla, en este trabajo nos referiremos a él con el 
nombre de Tejuela, que es el que se utiliza en las modernas publicaciones (Aratikos Arqueólogos, 2010; Palomino y Negredo, 2020). Este cementerio pertenece a lo que Padilla y Álvaro denominan como "grandes necrópolis", es decir, conjuntos de enterramiento de comunidades aldeanas estables con más de cien tumbas situadas en torno a un centro de culto (Padilla y Álvaro, 2010). En este sentido Tejuela cuenta con al menos 340 tumbas que rodean una pequeña iglesia y un edículo (Palomino y Negredo, 2020). El conjunto es muy similar a los cementerios burgaleses de Cuyacabras, Revenga o Regumiel y al soriano de Duruelo (Padilla y Álvaro, 2010 y 2013; López, Álvaro y Travé, 2016; Álvaro, Travé y López, 2018). Tejuela resulta un caso especial porque, a diferencia de la mayoría de los cementerios en roca, conservaba restos humanos en sus tumbas, estos se recogieron en las excavaciones y fueron estudiados por antropólogos. Aunque hay estudios sobre otras poblaciones altomedievales del norte peninsular (López, 2002; Candelas González et al., 2016; González Martín et al., 2016), Padilla y Álvaro (2010) señalan que de las comunidades aldeanas altomedievales se sabe muy poco en cuanto a la forma de vida de las familias, la gestión del espacio y los patrones de explotación del territorio circundante, mientras se conocen bien los cementerios. Sin embargo, y a este nivel, se podría añadir que tampoco se sabe mucho sobre la utilización de los mismos en el sentido de la distribución de las tumbas según sexo y edad y sus tipos y son estas cuestiones sobre las que se desarrolla el presente trabajo.

En las excavaciones de Alberto del Castillo (1972) se obtuvieron restos de no menos de 115 individuos de cuyo estudio se encargó el profesor Philippe du Souich, miembro del Laboratorio de Antropología Física de la Universidad de Granada (Du Souich, Botella y Ruiz, 1991). Tanto los trabajos realizados por este profesor como los diversos estudios posteriores sobre esta colección carecieron de información arqueológica detallada del cementerio y se limitaron a recoger los datos y teorías planteados en la publicación de Alberto del Castillo (Du Souich, Botella y Ruiz, 1991; Al Oumaoui, Jiménez-Brobeil y Du Souich, 2004; Maroto, 2004; Castillo, 2008; Jiménez-Brobeil et al., 2012; entre otros). La única información que acompañaba a cada esqueleto era el número de tumba en la que se había hallado y nunca se dispuso de plano del yacimiento, información sobre el tipo de enterramiento o su ubicación. En el año 2010 la Excma. Diputación de Burgos inició una puesta en valor del cementerio con una nueva excavación efectuada por el equipo de Aratikos Arqueólogos (Aratikos Arqueólogos, 2010; Palomino y Negredo, 2020). Este proyecto ha abierto una nueva colaboración entre arqueólogos y antropó- logos, una generación después de los pioneros, que permitirá un importante avance en el conocimiento de esta comunidad aldeana. La excavación y limpieza de más tumbas, el hallazgo de nuevos esqueletos, la ubicación sobre plano de los individuos excavados por Alberto del Castillo y la obtención de dataciones absolutas sobre estos, convierten a Tejuela en un referente clave dentro de los cementerios altomedievales del norte peninsular.

Con estos precedentes, el objetivo de este trabajo es intentar responder a varias preguntas surgidas a partir de los antiguos estudios: ¿hay algún patrón en la distribución de las tumbas?, ¿hay relación entre el tipo de tumba y su longitud con el sexo y edad del individuo enterrado en ella? y ¿qué cronología tienen los enterramientos?

\section{MATERIAL Y MÉTODOS}

En este trabajo se ha empleado únicamente información aportada por los esqueletos perfectamente ubicados en tumbas numeradas tanto de la excavación de Alberto del Castillo (1972) como de la de Aratikos Arqueólogos (2010). Los números de tumba que se emplean se refieren al inventario realizado en la última excavación (Aratikos Arqueólogos, 2010; Centeno, Negredo y Palomino, 2020). En total se incluyen datos de 109 individuos, 87 procedentes de la excavación de Alberto del Castillo y 22 de la del año 2010. Conviene tener en cuenta que en ambas excavaciones se encontraron tumbas vacías y que en la última se han documentado enterramientos que no se han excavado (Centeno, Negredo y Palomino, 2020).

Las variables analizadas en este trabajo son el sexo de los individuos, la edad, la estatura, el tipo de tumba y el tamaño de la misma. El sexo de los sujetos se ha determinado con base en los criterios habituales de morfología pélvica y craneal (Byers, 2005). Se han establecido tres categorías: varón, mujer y desconocido. Esta última engloba a los esqueletos a los que no puede determinarse el sexo, bien porque son subadultos en los que no se distinguen caracteres sexuales secundarios o adultos en mal estado conservación. La edad se ha estimado a partir de la maduración esquelética y dental, sínfisis del pubis, faceta esternal de las costillas y obliteración de las suturas craneales (Ubelaker, 1978; Krogman e Isçan, 1986; Scheuer y Black, 2000; Byers, 2005). Se han estimado en total 7 grupos: infantil entre 0 y 2 años, infantil entre 3 y 6 años, infantil entre 7 y 12 años, juvenil entre 13 y 20 años, adulto entre 21 y 40 años, maduro entre 41 y 60 años y senil con más de 61 años. Aunque la estatura de los esqueletos estudiados, se ha estimado por diversos 
procedimientos (Maroto, 2004), en este trabajo se ha utilizado el método de Olivier y Tissier de 1975. Este parámetro solo ha podido calcularse en los casos en los que se conservaban huesos largos completos. Respecto al tipo de tumba se han establecido cuatro tipos: antropomorfa, de "bañera", mixta y de lajas con base en las descripciones publicadas (Aratikos Arqueólogos, 2010). Entre las primeras se incluyen las calificadas como tales y las denominadas antropomorfas incipientes. No se incluyen los sarcófagos por no contar ninguno con restos óseos. En cuanto al tamaño se han establecido cuatro categorías: 1) con longitud menor de 1 metro; 2) con longitud comprendida entre $101 \mathrm{y}$ $130 \mathrm{~cm}$; 3) con longitud entre 131 y $160 \mathrm{~cm}$; y 4) cuya longitud supera los $160 \mathrm{~cm}$. Las dimensiones se han tomado de las publicadas por Aratikos Arqueólogos en 2010. Se han efectuado análisis estadísticos relacionando sexo y edad con tipo de tumba y tamaño de las mismas y con tamaño de las tumbas y estatura de los individuos contenidos en ellas. Se han empleado los test chi cuadrado $\left(\chi^{2}\right)$ y $t$ de Student y el coeficiente de correlación de Pearson (Schwartz, 1985). Todos los cálculos se han llevado a cabo con el programa estadístico SPSS versión 22 de IBM.

Las dataciones absolutas se han llevado a cabo en el Centro Nacional de Aceleradores (CNA) de Sevilla, perteneciente al Consejo Superior de Investigaciones Científicas, a partir de colágeno extraído de costillas de 11 esqueletos.

\section{DISTRIBUCIÓN GENERAL DEL CEMENTERIO}

En la Figura 1 se representa la planta del cementerio de Tejuela (Villanueva de Soportilla) según la última excavación llevada a cabo en el año 2010. La descripción detallada de las tumbas y su ubicación en el cementerio pueden consultarse en las recientes publicaciones sobre el yacimiento (Aratikos Arqueólogos, 2010; Palomino y Negredo, 2020). En este plano se encuentran marcadas con color las tumbas que han proporcionado restos humanos tanto de la excavación de Alberto del Castillo como de la de Aratikos Arqueólogos. Las tumbas sin color se encontraron vacías o no se han excavado ${ }^{1}$. En color azul se representan las sepulturas que contenían esqueletos de varones; en color naranja figuran las que albergaban esqueletos femeninos y en verde, las tumbas con individuos subadultos. Algunos enterramientos, marcados en color violeta, contenían restos esqueléticos en muy mal estado de conservación de adultos a

1 Han quedado 71 tumbas como reserva arqueológica (Centeno, Negredo y Palomino, 2020). los que no ha podido determinarse el sexo. Todas las tumbas tenían huesos pertenecientes a un solo esqueleto, aunque en algunos casos no era así. Por ejemplo, las tumbas número 134 y 320 contenían restos de un adulto de sexo indeterminable y de un subadulto. La tumba número 107 contenía un varón, colocado en decúbito supino, y huesos sueltos de una mujer y un niño. Un caso especial es la tumba 167 , cuyo tamaño $(56 \mathrm{~cm})$ es propio de un recién nacido y sin embargo contenía huesos sueltos pertenecientes a dos varones, una mujer y un subadulto. Los enterramientos de adultos se distribuyen de forma aleatoria por todo el yacimiento, lo que coincide con otros cementerios de esta época como los de Veranes o Marialba de la Ribera (Candelas González et al., 2016; González Martín et al., 2016) y solo se aprecia una concentración de tumbas, todas infantiles, en el lateral sur de la iglesia, fenómeno documentado en otras necrópolis castellanas (Padilla y Álvaro, 2008, 2010 y 2013; Candelas González et al., 2016). En otros cementerios medievales europeos es común el hallazgo de agrupaciones de tumbas con individuos perinatales o de muy corta edad bajo la línea de escorrentía del alero del tejado de las iglesias (Séguy, 1997; Craig-Atkins, 2014). Hay muchas interpretaciones al respecto (Gélis, 2006), principalmente sobre la situación social de los niños fallecidos sin bautizar, pero supone en alguna medida el reconocimiento de la identidad de estos niños dentro de la comunidad. La distribución tipológica de las tumbas en el cementerio está marcada por las características físicas del sustrato natural y así, por ejemplo, la mayoría de tumbas de lajas se ubica en el cuadrante noreste en una zona erosionada y colmatada con sedimento arenoso (Aratikos Arqueólogos, 2010; Centeno, Negredo y Palomino, 2020).

En principio, todas las sepulturas de Tejuela son individuales, con los sujetos yacentes en decúbito supino siguiendo el habitual ritual funerario cristiano (Ariès, 1992). Al no disponer de dibujos o fotografías detalladas de los hallazgos de las excavaciones de Alberto del Castillo no se puede precisar si las escasas tumbas con restos de más de un individuo corresponden a casos de reutilización o si bien los huesos podían proceder de otro enterramiento alterado y que por diversas circunstancias sufrió una dispersión de piezas. En la excavación más moderna (Aratikos Arqueólogos, 2010) sí se ha reflejado el hallazgo de restos esqueléticos sueltos diferentes al sujeto principal inhumado, piezas con toda probabilidad procedentes de enterramientos alterados (García Martínez, 2018; Martín-Alonso, 2018; Centeno, Negredo y Palomino, 2020). La tumba 167, con huesos sueltos de 5 individuos puede considerarse como un depósito de tumbas alteradas más que como un osario, dado que alguno de los individuos está representado por solo una pieza 


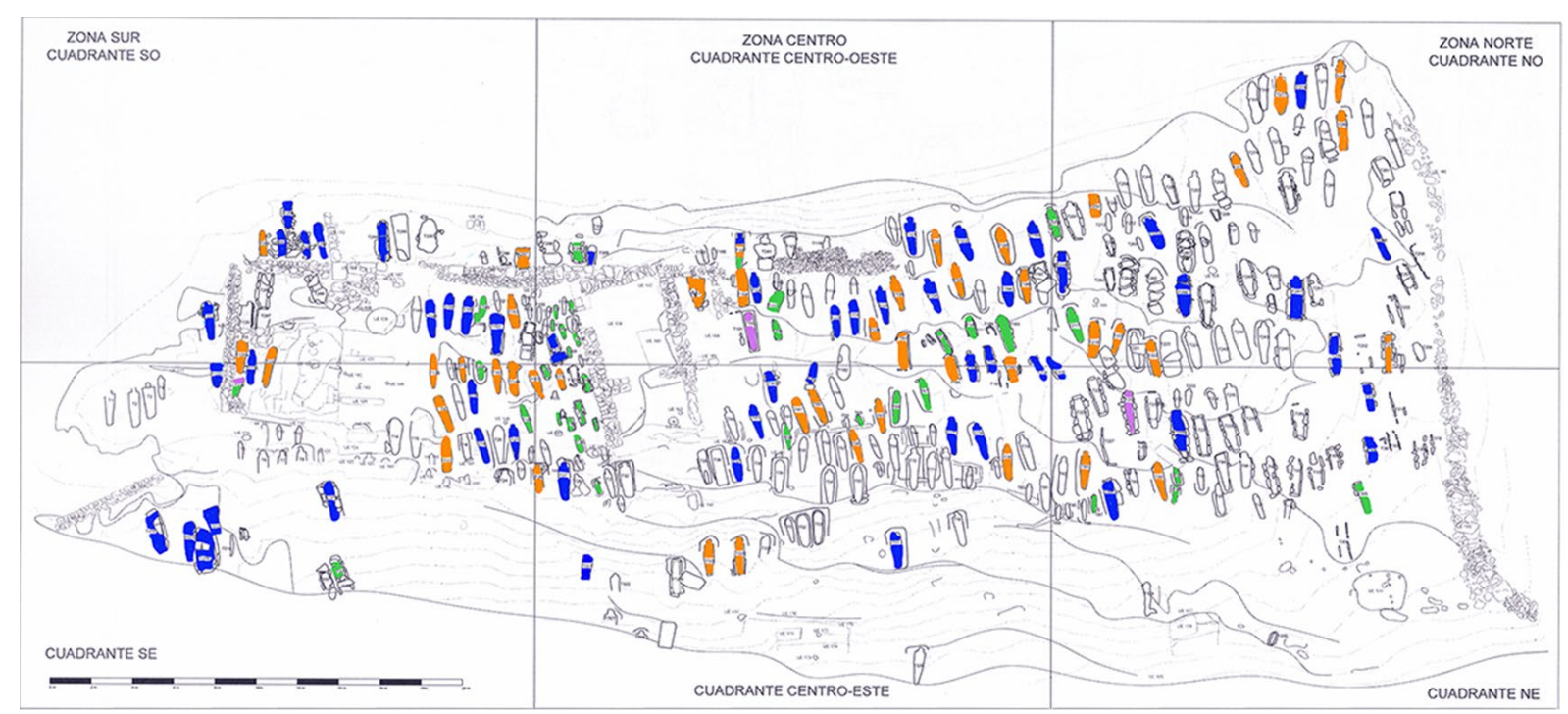

Figura 1. Distribución de los esqueletos con sexo y edad en el cementerio de Tejuela. Planta tomada de Aratikos Arqueólogos (2010) y modificada por los autores con la información antropológica. En color azul: varones; en color naranja: mujeres; en color violeta: adultos alofisos; en color verde: subadultos.

del esqueleto. Es posible que alguna tumba se reutilizara, pero no se ha hallado claramente un osario en el sentido de un depósito secundario al que se trasladaran esqueletos, más o menos completos, procedentes de su sepultura original. En otro cementerio medieval excavado por Alberto del Castillo, el de San Baudelio de Berlanga, sí se observa claramente una reutilización de las tumbas en el sentido de usarlas como posibles panteones familiares. Al enterrar a un individuo, se arrinconaban los huesos del depositado anteriormente y así se llega al caso de encontrar varios sujetos en una misma tumba (Maroto, 2004). También se han señalado reutilizaciones en el cementerio altomedieval de Veranes en Asturias (González Martín et al., 2016).

Es difícil establecer posibles relaciones familiares entre los individuos a partir de la posición de las tumbas en el cementerio. En la última excavación se señalan varias tumbas que por sus características y ubicación podrían pertenecer a miembros de una misma unidad familiar (Centeno, Negredo y Palomino, 2020). Así, por ejemplo, se destacan los números 51,52 y 53 , situadas en el cuadrante centro-este de la necrópolis, o las 1, 2, 3 y 4 en el cuadrante sureste (Fig. 1), pero desgraciadamente, no conservan restos esqueléticos que puedan compararse entre sí. Otros dos casos, el de la 120 y la 121 y el de la 253 y 254 , presentan una tumba con un adulto junto a una posi- blemente infantil por su tamaño, pero no se dispone de restos esqueléticos de los subadultos. Los estudios antropométricos de Philippe du Souich señalan que hay una cierta tasa de endogamia en el yacimiento lo que supondría que una buena parte de los individuos enterrados en él, tuvieran un mayor o menor grado de parentesco (Du Souich, Botella y Ruiz, 1991), pero no se han llevado a cabo análisis de ADN.

\section{DISTRIBUCIÓN DE TUMBAS POR SEXO Y EDAD}

Los 109 esqueletos estudiados suponen un total de 56 varones $(51,4 \%), 29$ mujeres $(26,6 \%)$ y 24 alofisos $(22,0 \%)$. El tipo de tumba se ha podido establecer en 106 casos que se distribuyen entre 37 antropomorfas (33,9\%), 18 de "bañera" (16,5\%), 24 mixtas $(22,6 \%)$ y 27 de lajas $(24,8 \%)$ pertenecientes a 54 varones, 28 mujeres y 24 alofisos. En las Figuras 2 y 3 se expone la distribución cruzada de ambas variables en la que se aprecia un reparto semejante entre ambos sexos, aunque con predominio masculino, en cuanto a tumbas antropomorfas; una mayoría de tipo bañera entre las mujeres y una cifra claramente superior de los tipos restantes entre los varones. Pero hay que tener en cuenta que las tumbas femeninas son casi la mitad que las masculinas y por tanto hay que analizar la distribución de tipos 
de tumbas dentro de cada sexo. En este caso (Fig. 4), los varones enterrados en tumbas antropomorfas suponen un $35,2 \%$, frente a un $42,8 \%$ de las mujeres, pero no hay significación estadística $\left(\chi^{2}=0,19 ; \mathrm{P}=\right.$ $0,66)$. En tumbas tipo bañera se hallan inhumados un $5,6 \%$ de varones y un $32,1 \%$ de mujeres y esta diferencia resulta muy significativa $\left(\chi^{2}=8,41 ; \mathrm{P}<\right.$ $0,001)$, es decir, no se debe al azar. En las tumbas de tipología mixta descansaban un 33,3\% de varones frente a un 7,1\% de mujeres y la diferencia resulta estadísticamente significativa $\left(\chi^{2}=5,51 ; \mathrm{P}=0,02\right)$. Por último, en tumbas de lajas se hallaron un 25,9\% de varones y un $17,8 \%$ de mujeres y en este caso sin significación estadística $\left(\chi^{2}=0,30 ; \mathrm{P}=0,58\right)$.

\begin{tabular}{|l|l|c|c|c|c|}
\hline \multicolumn{2}{|c|}{} & \multicolumn{3}{c|}{ Sexo } & \multirow{2}{*}{} \\
\cline { 3 - 6 } & Varón & Mujer & $\begin{array}{c}\text { Indetermi- } \\
\text { nable }\end{array}$ & Total \\
\hline \multirow{2nnyyyn}{*2}{ Tipo } & Antropomorfa & 19 & 12 & 6 & 37 \\
\hline & Bañera & 3 & 9 & 6 & 18 \\
\hline & Mixta & 18 & 2 & 4 & 24 \\
\hline Total & Lajas & 14 & 5 & 8 & 27 \\
\hline
\end{tabular}

Figura 2. Distribución de tumbas según tipo y sexo.

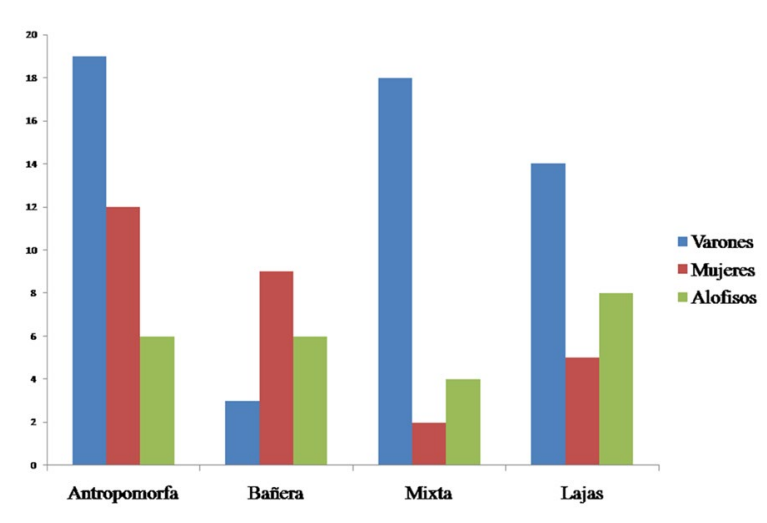

Figura 3. Distribución de tumbas según tipo y sexo de los esqueletos. Los alofisos son fundamentalmente subadultos (elaboración propia)

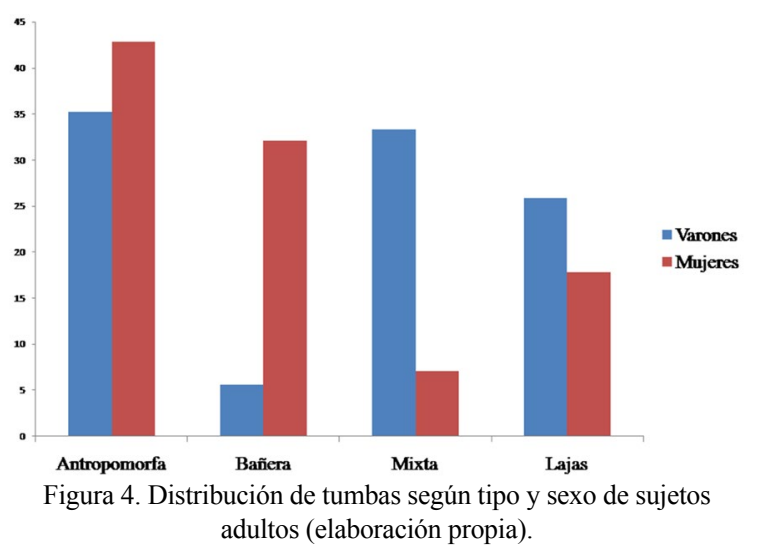

Alberto del Castillo describe que en las tumbas excavadas en la roca se alternaban las de tipo antropomorfo con las ovaladas o de bañera y lo atribuyó a una posible asociación de tipo de tumba y sexo del individuo inhumado en ella (Castillo, 1972). Concretamente, consideraba "probablemente femeninas" a las de bañera. Como se puede observar en los resultados obtenidos no hay diferencias significativas en la distribución por sexo de las tumbas antropomorfas y de lajas. Sin embargo, figura una mayoría de tipo bañera entre las mujeres y de tipo mixto entre los varones y esta distribución no se debe al azar. En este sentido, el profesor Del Castillo llevaba razón en que las tumbas de bañera son mucho más frecuentes entre las mujeres, pero el hallazgo de varones en ellas no significa que sean exclusivamente femeninas. Lo mismo puede aplicarse a las de tipología mixta, aunque en sentido inverso. Desgraciadamente, no podemos ni siquiera intuir las razones por las que se utilizó un tipo de tumba u otro para cada individuo enterrado en ella.

La edad ha podido estimarse en 108 casos que se distribuyen como sigue: doce sujetos entre 0 y 2 años $(11,0 \%)$; ocho entre los 3 y los 6 años $(7,3 \%)$; cinco entre 7 y 12 años (4,6 \%); cuatro juveniles $(3,7 \%)$; cincuenta adultos $(45,9 \%)$; veintiséis maduros $(23,9 \%)$ y tres seniles $(2,8 \%)$. En las Figuras 5 y 6 se puede observar la distribución de los casos según edad y en los que se ha determinado el tipo de tumba (105 casos). No hay una asociación clara entre estas dos variables con la excepción de las tumbas tipo bañera que, entre los individuos infantiles, son propias de los fallecidos antes de cumplir los 2 años $(\mathrm{P}=0,01)$.

\begin{tabular}{|c|c|c|c|c|c|c|c|c|}
\hline & \multicolumn{7}{|c|}{ Edad } \\
\hline & & $\begin{array}{l}\mathcal{O} \\
\stackrel{1}{\Xi} \\
\end{array}$ & 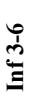 & $\Xi$ & 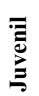 & $\frac{\stackrel{\theta}{E}}{\frac{E}{2}}$ & & 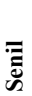 \\
\hline Tipo & Antropomorfa & 1 & 3 & 2 & 1 & 22 & 7 & 1 \\
\hline & Bañera & 5 & 0 & 1 & 0 & 9 & 2 & 0 \\
\hline & Mixta & 2 & 1 & 2 & 2 & 7 & 9 & 1 \\
\hline & Lajas & 4 & 4 & 0 & 0 & 11 & 7 & 1 \\
\hline Total & & 12 & 8 & 5 & 3 & 49 & 25 & 3 \\
\hline
\end{tabular}

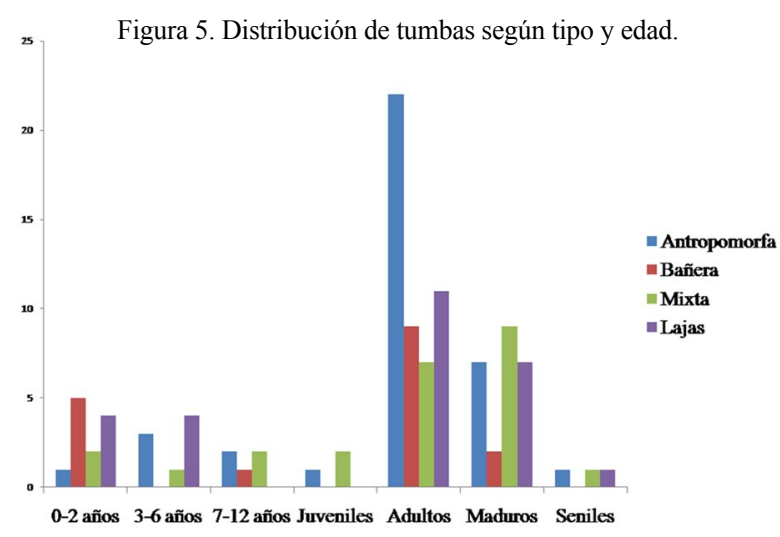

Figura 6. Distribución de tumbas según tipo y grupo de edad (elaboración propia). 
En relación con el tipo de tumba y la edad de los individuos, el profesor Del Castillo planteó que las tumbas "infantiles y de muchacho suelen ser ovaladas" (Castillo, 1972). En el total de 68 tumbas tipo bañera documentadas en el yacimiento de Tejuela (Aratikos Arqueólogos, 2010), 29 de ellas (42,6 \%) tienen una longitud inferior a un metro. En la necrópolis de Revenga (Padilla y Álvaro, 2013) la mayoría de las tumbas pequeñas son de tipo bañera $(47,9 \%)$, e igual se aprecia en las plantas de los cementerios de Duruelo y Cuyacabras (Padilla y Álvaro, 2008 y 2010). Muchas de las tumbas de Tejuela, inferiores a un metro de longitud, se encontraron vacías, bien por la mala conservación de los extremadamente delicados huesos de los niños muy pequeños o porque si lo conservado estaba reducido a esquirlas, estas no se recogieron. Los restos óseos recuperados de niños aparecen en tumbas de diversa tipología, pero entre las que están excavadas en la roca no se debe al azar el que la mayoría de los muy pequeños se encuentre en tumbas de tipo bañera. En cuanto a las 51 tumbas cuya longitud se encuentra entre los 100 y $150 \mathrm{~cm}$, que fueron denominadas como de "muchacho" por el profesor Alberto del Castillo, solo un 25,5\% pertenecen al tipo bañera y el más frecuente es el de lajas (Palomino y Negredo, 2020). Tan solo una de estas tumbas tipo bañera conservaba restos óseos pertenecientes a un individuo infantil entre 7 y 12 años. Como se puede ver, con la excepción de una mayoría de tumbas de tipo bañera entre los individuos infantiles más pequeños, no figura ningún patrón de tipo de enterramiento entre los sujetos subadultos. Desafortunadamente, no es posible determinar con precisión el sexo de los esqueletos infantiles, lo que impide observar si pudieran existir diferencias entre tumbas de niñas y niños.

\section{TAMAÑO DE TUMBA, EDAD Y ESTATURA}

En cuanto al tamaño de las tumbas solo 91, que contengan restos esqueléticos, permiten la medición completa de su longitud. Las más dañadas son las de lajas y las mixtas, donde es común la pérdida de algunos elementos que impiden el registro correcto de este parámetro. Las tumbas con longitud inferior a un metro son en total $15(16,5 \%)$ y las que miden entre $101 \mathrm{y} 130 \mathrm{~cm}$ son siete $(7,7 \%)$. Cinco tumbas $(5,5 \%)$ se encuentran entre 131 y $160 \mathrm{~cm}$, y las 64 restantes $(70,3 \%)$ superan esta medida. En las Figuras 7 y 8 se expone la distribución cruzada de edad y tamaño de tumba.

Como puede apreciarse, en las tumbas inferiores a un metro de largo se encuentran todos los niños menores de 2 años y tres de la siguiente categoría de edad (3-6 años). Entre los primeros hay que señalar que 5 individuos, todos recién nacidos y perinatales (meno- res de 2 meses), se hallaron en tumbas de menos de 60 $\mathrm{cm}$ de longitud. Cuatro niños de 4 a 12 meses de edad se hallaron en tumbas con longitud entre 61 y $80 \mathrm{~cm}$; tres niños, de uno a 2 años de edad yacían en tumbas de más de $81 \mathrm{~cm}$ junto con los tres niños restantes, todos con edad entre los 3 y 5 años.

\begin{tabular}{|l|c|c|c|c|c|}
\hline \multirow{2}{*}{} & \multicolumn{4}{|c|}{ Tamaño } & \multirow{2}{*}{ Total } \\
\cline { 2 - 5 } & $<\mathbf{1}$ & $\mathbf{1 - 1 . 3}$ & $\mathbf{1 . 3 - 1 . 6}$ & $>\mathbf{1 . 6}$ & \\
\hline Inf 0-2 años & 12 & 0 & 0 & 0 & 12 \\
\hline Inf 3-6 años & 3 & 5 & 0 & 0 & 8 \\
\hline Inf II (7-12 años) & 0 & 1 & 4 & 0 & 5 \\
\hline Juvenil & 0 & 1 & 0 & 1 & 2 \\
\hline Adulto & 0 & 0 & 1 & 38 & 39 \\
\hline Maduro & 0 & 0 & 0 & 22 & 22 \\
\hline Senil & 0 & 0 & 0 & 3 & 3 \\
\hline Total & 15 & 7 & 5 & 64 & 91 \\
\hline
\end{tabular}

Figura 7. Distribución de tumbas según edad y longitud.

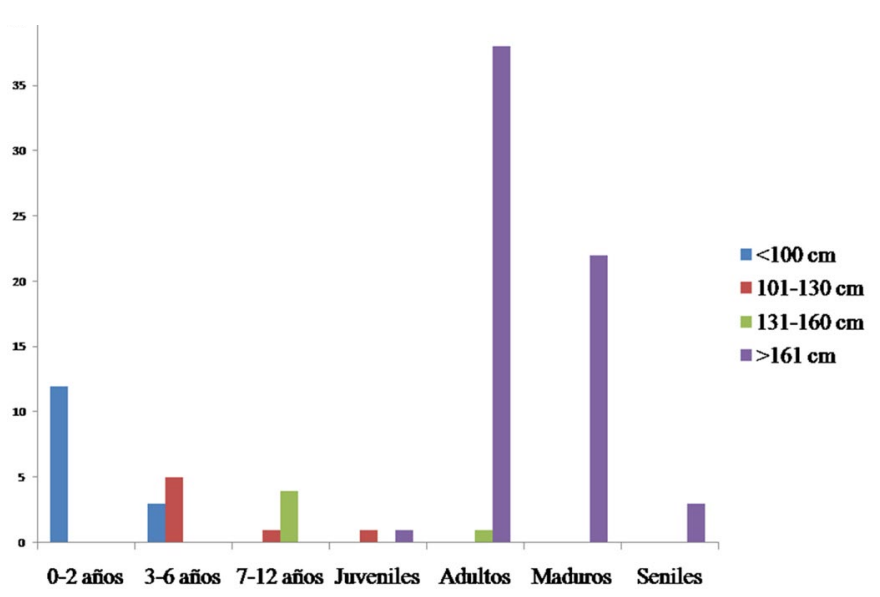

Figura 8. Distribución de tumbas según longitud y grupo de edad (elaboración propia).

En las tumbas de 101 a $130 \mathrm{~cm}$ se encontraron niños entre 3 y 7 años y un individuo juvenil de 13 años. En el siguiente tamaño de tumbas, de 131 a $160 \mathrm{~cm}$, se ubican los niños restantes, todos mayores de 7 años, $\mathrm{y}$ un sujeto adulto, de sexo femenino, con una estatura de $151 \mathrm{~cm}$. En las tumbas más largas yacían un sujeto juvenil de sexo masculino y los restantes individuos adultos, maduros y seniles. Como otros autores (Castillo, 1972; Padilla y Álvaro, 2013; Palomino y Negredo, 2020) han establecido una categoría de 101 a $150 \mathrm{~cm}$, de cara a posibles comparaciones se aclara que entre 131 y $150 \mathrm{~cm}$ solo figuran niños entre 7 y 11 años y un juvenil de 13 . 
Se ha podido calcular la estatura de 30 varones y 24 mujeres. Los primeros tienen tallas entre 156 y 177 $\mathrm{cm}$ con un valor medio de $167,6 \pm 4,23 \mathrm{~cm}$. Las segundas presentan valores entre 150 y $162 \mathrm{~cm}$ con una media de $156,5 \pm 3,11 \mathrm{~cm}$. La longitud de las tumbas con esqueletos masculinos, con 25 casos conservados, oscila entre 165 y $200 \mathrm{~cm}$, con una media de 180,4 $\pm 9,5 \mathrm{~cm}$. Las tumbas con esqueletos femeninos que conservan completa su longitud son 20 y oscilan entre los 160 y los $185 \mathrm{~cm}$, con una cifra media de 176,4 \pm $7,1 \mathrm{~cm}$. Existe correlación significativa entre la estatura y la longitud de la tumba $(0,511$ de Pearson con $\mathrm{P}<0,001)$ y diferencia estadísticamente significativa entre los valores medios masculino y femenino $(t=$ $4,48 ; \mathrm{P}<0,001)$.

Como se ha visto en cuanto a la edad de los individuos y el tamaño de las tumbas, todos los niños pequeños menores de 2 años, junto con tres individuos fallecidos entre los 3 y 5 años, se encuentran en tumbas inferiores al metro de longitud. Esto resulta normal si tenemos en cuenta que, según las curvas de crecimiento de niños españoles actuales de la Fundación Faustino Orbegozo (Sobradillo et al., 2004), un metro es la talla media para los 4 años de edad. También es lógico que las tumbas de menos de $60 \mathrm{~cm}$ de longitud contengan recién nacidos o perinatales puesto que la talla media al término del embarazo es de unos $50 \mathrm{~cm}$ (Sobradillo et al., 2004). Hay que usar estas tablas de niños actuales con precaución porque si bien la estatura media de los varones y mujeres adultos de Tejuela, estimada por el método de Olivier-Tissier, resulta similar a la media española del siglo XX (Martínez y Puche, 2011), los patrones de crecimiento de los niños no tienen por qué coincidir. Hay que tener en cuenta que en la velocidad del crecimiento influyen diversos factores de índole socioeconómica, como son la nutrición (por ejemplo, las restricciones alimenticias producen retraso), el hábitat (diferencias ciudad-campo) y la clase social e ingresos económicos de las familias (Valls, 1985).

En tumbas con longitudes entre 101 y $130 \mathrm{~cm}$ se encontraron cinco individuos de 3 a 6 años, uno de 7 a 12 años y un juvenil. La estatura media de los niños y niñas españoles actuales de 8 a 9 años, es precisamente de $130 \mathrm{~cm}$, por lo que las tumbas coinciden con la talla que se esperaría para la edad de estos niños. Solo figura la excepción del sujeto juvenil, fallecido en torno a los 13 años y con posible sexo femenino. Se le ha estimado una estatura (Scheuer y Black, 2000) en torno a $125-130 \mathrm{~cm}$, similar a la media de los 8-9 años de edad. En niñas actuales (Sobradillo et al., 2004) el percentil más bajo para 12-13 años es de $135 \mathrm{~cm}$. Esta joven sería muy pequeña para su edad y por ello pudo inhumarse en una tumba con un tamaño más propio de niños.
Las tumbas entre 131 y $160 \mathrm{~cm}$ albergan cuatro individuos entre 7 y 12 años. La talla media de niños y niñas actuales a los 12 años es de $150 \mathrm{~cm}$ (Sobradillo et al., 2004). También, como se expone más arriba, una mujer de $151 \mathrm{~cm}$ de estatura, es el único sujeto adulto enterrado en una tumba de este tamaño. En cualquier caso, sería muy arriesgado estimar con precisión la edad del posible individuo inhumado en una tumba que se descubriera vacía.

En las tumbas con longitudes superiores a $160 \mathrm{~cm}$ solo se encuentran adultos y un joven, posiblemente masculino, de 13 años. Dentro de estas tumbas de adultos, como se ha visto más arriba, existe correlación entre la estatura del individuo inhumado y la longitud máxima de la tumba, pero dada la variabilidad de tamaños, no sería fiable determinar el sexo del posible sujeto enterrado en una tumba que no conservara el esqueleto.

\section{DATACIONES ABSOLUTAS}

Las dataciones absolutas que aquí se presentan (Figs. 9 y 10) proceden de 11 esqueletos exhumados en las excavaciones de Alberto del Castillo. Coincidiendo con la redacción de este trabajo se han procesado más muestras obtenidas de las tumbas excavadas en 2010 cuyos resultados se esperan próximamente. También se hallan en proceso de datación muestras procedentes de la necrópolis del Castillo de Palacios de la Sierra (Burgos), por lo que se estima que los resultados que se obtengan aportarán una información fundamental en el tema de la cronología de los cementerios medievales burgaleses. Las 11 muestras no se seleccionaron con criterios arqueológicos sino con base en la calidad del colágeno preservado, obtenido para llevar a cabo análisis de isótopos estables enfocados al estudio de la salud y la alimentación de esta población.

Como puede observarse en las Figuras 9 y 10, las dataciones calibradas a $\pm 2 \sigma$ corresponden a un periodo que se extiende desde el último tercio del siglo VIII a comienzos del XI con una ocupación centrada en el siglo X. Obviamente, estas dataciones fechan el fallecimiento de los individuos de cuyos esqueletos se han tomado las muestras. Si se considera que aparentemente no hubo reutilizaciones, pueden aplicarse, con la debida cautela, a la cronología de la realización de las tumbas. En cualquier caso, no informan sobre los momentos de fundación y abandono del poblado de Tejuela.

Los resultados obtenidos no coinciden con la hipótesis de Alberto del Castillo quien proponía fechas del IX-X para el inicio de la puesta en uso del cementerio, que vinculaba con un proceso de repoblación que podría iniciarse durante el reinado de Ramiro I (Casti- 


\begin{tabular}{|c|c|c|c|c|c|c|c|}
\hline Muestra & Tumba & Sector & Tipo & Código & Data Conv & Cal $68.2 \%$ & Cal $95.4 \%$ \\
\hline V-1 & 147 & SO & $\begin{array}{l}\text { Antropomorfa } \\
\text { incipiente }\end{array}$ & CNA 4426 & $1180 \pm 30$ & $777-887$ & $730-951$ \\
\hline $\mathrm{V}-3$ & 48 & $\mathrm{CE}$ & Bañera & CNA 4424 & $1170 \pm 30$ & $777-893$ & $771-965$ \\
\hline $\mathrm{V}-28$ & 19 & SE & Bañera & CNA 4586 & $1160 \pm 30$ & $778-944$ & $773-968$ \\
\hline V-31 & 153 & SO & $\begin{array}{l}\text { Antropomorfa } \\
\text { incipiente }\end{array}$ & CNA 4587 & $1150 \pm 30$ & $778-967$ & $776-971$ \\
\hline V-4 & 65 & $\mathrm{CE}$ & $\begin{array}{l}\text { Antropomorfa } \\
\text { incipiente }\end{array}$ & CNA 4425 & $1130 \pm 30$ & 888-969 & $777-988$ \\
\hline $\mathrm{V}-22$ & 222 & NE/NO & Antropomorfa & CNA 4584 & $1130 \pm 30$ & 888-969 & $777-988$ \\
\hline $\mathrm{V}-27$ & 41 & $\mathrm{CE}$ & $\begin{array}{l}\text { Antropomorfa } \\
\text { incipiente }\end{array}$ & CNA 4588 & $1130 \pm 30$ & $888-969$ & $777-988$ \\
\hline $\mathrm{V}-2$ & 186 & $\mathrm{CE} / \mathrm{CO}$ & Lajas & CNA 4423 & $1120 \pm 30$ & 893-970 & 778-995 \\
\hline $\mathrm{V}-7$ & 188 & $\mathrm{CE} / \mathrm{CO}$ & Lajas & CNA 4427 & $1120 \pm 30$ & $893-970$ & $778-995$ \\
\hline V-19 & 123 & $\mathrm{CO}$ & Antropomorfa & CNA 4428 & $1100 \pm 30$ & $898-985$ & $887-1013$ \\
\hline $\mathrm{V}-23$ & 121 & $\mathrm{CO}$ & Antropomorfa & CNA 4585 & $1080 \pm 30$ & 901-996 & 894-1018 \\
\hline
\end{tabular}

Figura 9. Dataciones absolutas, tipos de tumba, sector del cementerio y códigos de muestra. Calibraciones a 1 y 2 sigmas según programa OxCal 4.3 (Reimer et al., 2013). SO: cuadrante suroeste; SE: cuadrante sureste; CE: cuadrante centro este; CO: cuadrante centro oeste; NE: cuadrante noreste; NO: cuadrante noroeste.

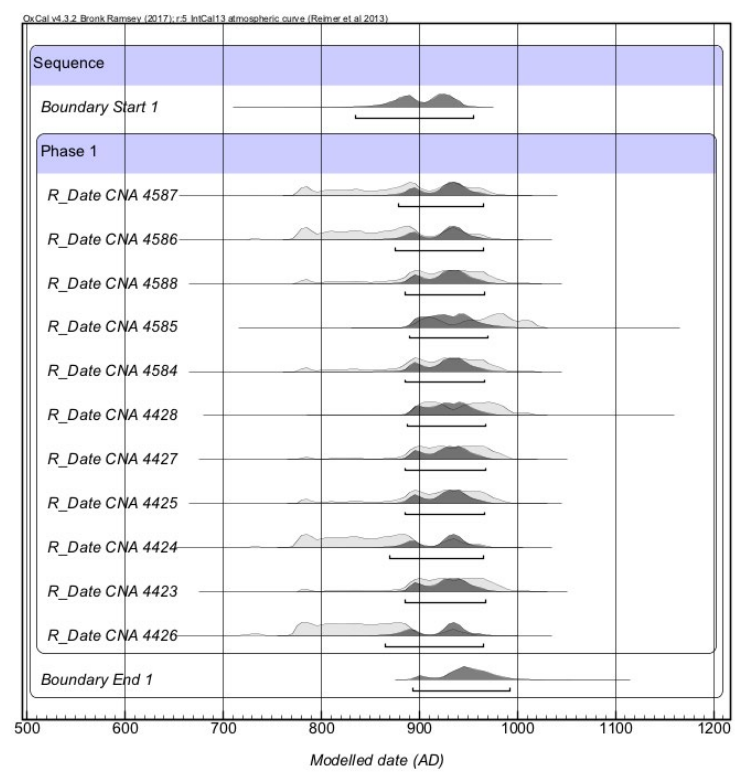

Figura 10. Representación gráfica de la estadística bayesiana de las dataciones absolutas (Bronk Ramsey, 2017). Curva atmosférica Intcal13 (Reimer et al., 2013) (elaborada por R. Martínez).

1lo, 1972). Del Castillo consideraba que las tumbas de bañera eran las más antiguas, seguidas de las antropomorfas, que situaba entre los siglos X y XII, y que las de lajas serían las más modernas. Este procedimiento de datación, basado en la tipología de los enterramientos, fue también utilizado por Cantera y Andrío (1991), quienes fecharon Tejuela entre finales del siglo IX y mediados del XII. Una mayoría de autores consideran que la tipología no sirve para establecer la cronología de las tumbas e insisten en la necesidad de obtener dataciones absolutas (Martín Viso, 2012 y 2014; Hernández et al., 2013; López, Álvaro y Travé, 2016). Las dataciones obtenidas en este estudio coinciden con las estimaciones modernas, que van del VIII al XII, según fuentes documentales (Moratinos, 2020) y hallazgos materiales (Centeno, Negredo y Palomino, 2020). Con relación a otros cementerios del norte peninsular, Tejuela es más moderno que el cercano de San Miguele en Álava (Gil y Sáenz, 2001) y más o menos contemporáneo del de Olèrdola (Molist y Bosch, 2012).

Once muestras no son suficientes para llevar a cabo un estudio pormenorizado de las fases de utilización del cementerio. Sí se puede comentar que las obtenidas no están en contra de que las tumbas más antiguas fueran las situadas en las inmediaciones de la iglesia y el edículo (Palomino y Negredo, 2020), como es el caso de la 147, la 48 o la 19; sin embargo, no puede trazarse un esquema de las posibles fases con base en las tumbas restantes. Es interesante constatar que dos tumbas contiguas, la 186 y 188 , son contemporáneas. En cuanto a las opiniones de Alberto del Castillo de que las tumbas marcaran una cronología, es cierto que dos de las más antiguas (del VIII-IX) son de bañera y entre las más modernas, del IX-X, se encuentran dos de lajas, pero esto puede ser una mera casualidad y más cuando en Tejuela el tipo de tumba está claramente condicionado por las características del sustrato rocoso.

De todos modos y como se comentó al comienzo de este texto, los autores no somos quienes para discutir estos resultados que simplemente exponemos para el conocimiento de los especialistas. 


\section{CONCLUSIONES}

El ejemplo de los proyectos de investigación llevados a cabo en el cementerio de Tejuela demuestra la necesidad de la estrecha colaboración entre arqueólogos y antropólogos desde el inicio del trabajo de campo y no solo para los estudios de laboratorio. Con la excepción de las dataciones, que se han efectuado recientemente, los resultados de este trabajo se podrían haber obtenido hace casi 40 años de haberse producido una colaboración entre los especialistas. Cada investigador trabajaba de forma independiente sin cotejar sus datos y sin llegar a elaborarlos de forma conjunta. Los antropólogos han limpiado, restaurado y analizado unos esqueletos de los que no tenían más información que un número de tumba. Por otro lado, los arqueólogos se quejaban de que no podían decir nada del uso del cementerio porque no tenían información sobre el sexo y edad de los esqueletos hallados y más en el caso de Tejuela, donde sí se habían conservado restos humanos.

En Tejuela no se han documentado casos claros de reutilización de tumbas y los pocos huesos sueltos hallados con esqueletos más o menos completos parecen proceder de alteraciones de otras tumbas. Al no figurar un antropólogo en el equipo excavador, no pudo llevarse a cabo en el terreno una posible vinculación de estos huesos sueltos a tumbas concretas. No hay un patrón de distribución de tumbas con la excepción de una mayoría de enterramientos de niños de muy corta edad ubicados a lo largo de un lateral de la iglesia.

En cuanto a la distribución de tipos de tumbas por sexo y edad, solo puede asegurarse que la mayoría de las de tipo bañera aparece asociada con esqueletos femeninos o niños muy pequeños. En cualquier caso, no puede establecerse con certeza el posible sexo de un individuo por el tipo de tumba. Hay una clara relación entre la longitud de la tumba y la edad del individuo, pero en ningún caso pueden considerarse como de adolescentes o juveniles las sepulturas con longitudes entre 101 y $150 \mathrm{~cm}$ tal como proponía Alberto del Castillo (1972). Salvo un caso de una joven de talla muy baja, los esqueletos hallados son de niños menores de 12 años. En el caso de los adultos, están correlacionadas la longitud de la tumba con la estatura del individuo inhumado en ella pero, al ser las tumbas bastante más largas que la talla del sujeto, tampoco puede precisarse el sexo del individuo que pudiera haberse depositado en una tumba que se haya descubierto vacía.

En cuanto al periodo de utilización del cementerio, de momento solo puede asegurarse que se centra en el siglo $X$ con una calibración a $\pm 2 \sigma$ entre el último tercio del siglo VIII y comienzos del XI. En general estas dataciones denotarían un uso del cementerio en fechas anteriores a las propuestas por Alberto del Castillo (1972) para la fundación del enclave.

Este trabajo es una aportación al estudio de los cementerios altomedievales del norte peninsular y, en especial, a los que presentan tumbas excavadas en la roca. Su principal contribución es reforzar el yacimiento de Tejuela (Villanueva de Soportilla) como un referente clave en el estudio de las comunidades campesinas altomedievales tanto por la conservación de las estructuras funerarias como por la de un buen número de los esqueletos inhumados en ellas.

\section{AGRADECIMIENTOS}

Los autores quieren manifestar su agradecimiento a D. Ángel Palomino y Dña. María Negredo por confiarles el estudio de los restos humanos de este yacimiento y hacerles partícipes del proyecto de puesta en valor del mismo. De igual modo, quieren dar las gracias a D. Rafael Martínez de la Universidad de Granada por la elaboración de los resultados de las dataciones absolutas.

\section{BIBLIOGRAFÍA}

Al Oumaoui, I., Jiménez-Brobeil, S. A. y Du Souich, Ph. (2004). "Markers of activity patterns in some populations of the Iberian Peninsula". International Journal of Osteoarchaeology, 14, pp. 343-358. DOI: https://doi.org/10.1002/oa.719

Álvaro Rueda, K., Travé Allepuz, E. y López Pérez, M. D. (2018). "Excavaciones arqueológicas en el yacimiento altomedieval de Revenga: nuevos datos para el conocimiento de los espacios de hábitat altomedieval en el alto Arlanza (Burgos)". Territorio, Sociedad y Poder, 13, pp. 5-21. DOI: https://doi.org/10.17811/tsp.13.2018.5-21

Andrío Gonzalo, J. (1987). "Formas de enterramientos medievales en los valles del Ebro y Duero". II Congreso de Arqueología Medieval Española. Madrid: Comunidad de Madrid, T III, pp. 273-286.

Aratikos Arqueólogos. (2010). Excavación arqueológica en la necrópolis de Santa María de Tejuela, Villanueva Soportilla (Bozoó, Burgos). Informe técnico. Burgos: Diputación de Burgos.

Ariès, Ph. (1992). El hombre ante la muerte. Madrid: Taurus.

Bolòs, J. y Pagès, M. (1982). "Les sepultures excavades a la roca". Acta historica et archaeologica mediaevalia, 3, pp. 59-103.

Bronk Ramsey, C. (2017). "Methods for summarizing radiocarbon datasets". Radiocarbon, 59, pp. 1809-1833. DOI: https://doi.org/10.1017/rdc.2017.108

Byers, S. (2005). Introduction to forensic Anthropology. Boston: Allyn and Bacon.

Candelas González, N., Núñez Cantalapiedra, A., Rascón Pérez, J., Cambra-Moo, O., Muñoz Villarejo, F., Campo- 
manes Alvaredo, E., Gutiérrez González, J. A. y González Martín, A. (2016). "Características paleodemográficas de la población recuperada del cementerio de Marialba de la Ribera (Villaturiel, León, España) (s. IV-XIII)”. Munibe. Antropologia-Arkeologia, 67, pp. 151-166. DOI: https://doi.org/10.21630/maa.2016.67.07

Cantera Burgos, F. y Andrío Gonzalo, J. (1991). Historia Medieval de Miranda de Ebro. Burgos: Fundación Cultural Profesor Cantera Burgos.

Castillo, A. del. (1970). "Cronología de las tumbas llamadas olerdolanas". XI Congreso Nacional de Arqueología. Zaragoza: Universidad de Zaragoza, pp. 835-845.

Castillo, A. del. (1972). Excavaciones altomedievales en las provincias de Soria, Logroño y Burgos. Madrid: Ministerio de Educación y Ciencia.

Castillo González, C. (2008). Evolución de los estados de salud-enfermedad de poblaciones medievales del Alto Ebro y Alto Duero. Tesis Doctoral. Universidad de Granada.

Centeno Cea, I., Negredo García, M. y Palomino Lázaro, A. L. (2020). "El yacimiento arqueológico. Análisis y resultados de las intervenciones arqueológicas". En: Palomino, A. y Negredo, M. (Coords.). La comunidad aldeana de Tejuela en época medieval. Arqueología funeraria y doméstica en el Alto Valle del Ebro. Burgos: Diputación de Burgos, pp. 67-137.

Craig-Atkins, E. (2014). "Eavesdropping on short lives: eaves-drip burial and the differential treatment of children one year of and under in early Christian cemeteries". En: Hadley, D. M. y Hemer, K. A. (Eds.). Medieval childhood. Archaeological approaches. Oxford: Oxbow, pp. 95-113.

Du Souich, Ph., Botella, M. y Ruiz, L. (1991). “Antropología de la población medieval de Villanueva de Soportilla (Burgos)". Antropología y Paleoecología Humana, 6, pp. $57-83$.

García Martínez, V. (2018). Estudio antropológico en Villanueva Soportilla. Caracteres físicos y patologías. Trabajo fin de máster. Universidad de Granada.

Gélis, J. (2006). Les enfants des limbes. Mort-nés et parents dans l'Europe chretiénne. Paris: Audibert.

Gil Zubillaga, L. y Sáenz de Urturi, F. (2001). San Miguele. La necrópolis tardorromana, tardoantigua y altomedieval de San Miguele (Molinilla, Álava). Vitoria, Álava: Diputación Foral de Álava.

González Martín, A., Rascón Pérez, J., Cambra-Moo, O., Pimentel de Francisco, G. y Campo Martín, M. (2016). "Estudio poblacional del cementerio medieval de Veranes (Gijón, Asturias): estado de la cuestión”. En: Quirós Castillo, J. A. (Dir.). Demografia, Paleopatologías y desigualdad social en el Noroeste peninsular en época medieval. Bilbao: Universidad del País Vasco, pp. 83-95.

Härke, H. (2001). "Cemeteries as places of power". En: De Jong, M., Theuws, F. y van Rhijn, C. (Eds.). Topographies of power in the Early Middle Ages. The Transformation of the Roman World, 6. Leiden: Brill, pp. 9-30.

Hernández Barreña, D., Alonso Gavilán, G., Bécares Pérez, J. y Martín Viso, I. (2013). “Análisis geoarqueológico de las tumbas de El Encinar excavadas en el granito de Al- deadávila de la Ribera, Salamanca: contextualización y protocolo de trabajo". Studia Geologica Salmanticensia, 49 (2), pp. 87-134.

Jiménez-Brobeil, S. A., Roca-Rodríguez, M. G., Al Oumaoui, I. y Du Souich, Ph. (2012). "Vertebral pathologies and related activity patterns in two Mediaeval populations from Spain". Collegium Antropologicum, 36 (3), pp. 1019-1025.

Krogman, W. M. e Isçan, M. Y. (1986). The human skeleton in forensic medicine. Springfield: Charles C. Thomas.

López, B. (2002). Las poblaciones del Antiguo Reino de León: Antropología, Paleodemografía, Paleopatología. León: Universidad de León.

López Pérez, M. D., Álvaro Rueda, K. y Travé Allepuz, E. (2016). "Rock-cut cemeteries and settlement processes at the Upper Arlanza Basin (Burgos, Spain): a Late Antique and Early Medieval landscape analysis". Zephyrus, LXXVIII, pp. 173-191.

Maroto Benavides, R. M. (2004). Antropología de las poblaciones femeninas medievales del Alto Ebro y Alto Duero. Granada: Universidad de Granada.

Martín-Alonso, J. F. (2018). Patrones de actividad en una población altomedieval: Sta. María de Tejuela. Trabajo fin de máster. Granada: Universidad de Granada.

Martín Viso, I. (2007). "Tumbas y sociedades locales en el centro de la Península Ibérica en la Alta Edad Media: el caso de la comarca de Riba Côa (Portugal)". Arqueología y Territorio Medieval, 14, pp. 21-48. DOI: https:// doi.org/10.17561/aytm.v14i0.1503

Martín Viso, I. (2012). "Enterramientos, memoria social y paisaje en la Alta Edad Media: propuestas para un análisis de las tumbas excavadas en roca en el centro-oeste de la Península Ibérica”. Zephyrus, LXIX, pp. 165-187.

Martín Viso, I. (2014). “¿Datar tumbas o datar procesos? A vueltas con la cronología de las tumbas excavadas en roca en la Península Ibérica”. Debates de Arqueología Medieval, 4, pp. 29-65.

Martínez Carrión, J. M. y Puche Gil, J. (2011). "La evolución de la estatura en Francia y en España, 1770-2000. Balance historiográfico y nuevas evidencias". Dynamis, 31 (2), pp. 429-452.

DOI: https://doi.org/10.4321/s0211-95362011000200009

Molist Capella, N. y Bosch Casadevall, J. (2012). "El cementeri medieval de Sant Miquel d' Olèrdola (Olèrdola, Alt Penedès)". En: Molist, N. y Ripoll, G. (Eds.). Arqueologia funeraria al nord-est peninsular (segles VI-XII). Barcelona: Museu d'Arqueologia de Catalunya-Olèrdola, pp. 469-494.

Moratinos García, M. (2020). "Contexto historico. Referencias documentales escritas". En: Palomino, A. y Negredo, M. (Coords.). La comunidad aldeana de Tejuela en época medieval. Arqueología funeraria y doméstica en el Alto Valle del Ebro. Burgos: Diputación de Burgos, pp. 35-47.

Olivier, G. y Tissier, H. (1975). "Détermination de la stature et de la capacité cranienne". Bulletin et Mémoires de la Societé d'Anthropologie de Paris, 2 (XIII), pp. 1-11. DOI: https://doi.org/10.3406/bmsap.1975.1794 
Padilla, J. I. y Álvaro-Rueda, K. (2008). "El despoblado altomedieval de Cuyacabras (Burgos): Realidad, principios y argumentos". Acta histórica et archaeologica mediaevalia, 29, pp. 575-604.

Padilla, J. I. y Álvaro-Rueda, K. (2010). "Necrópolis rupestres y el poblamiento altomedieval en el alto Arlanza (Burgos)”. En la España Medieval, 33, pp. 259-294.

Padilla, J. I. y Álvaro-Rueda, K. (2013). “Asentamientos altomedievales del Alto Arlanza (Burgos). El despoblado medieval de Revenga". Pyrenae, 44 (1), pp. 11-41.

Palomino Lázaro, Á. L. y Negredo García, M. (Coords.) (2020). La comunidad aldeana de Tejuela en época medieval. Arqueología funeraria y doméstica en el Alto Valle del Ebro. Burgos: Diputación de Burgos.

Quirós Castillo, J. A. (2006). "La génesis del paisaje medieval en Álava: la formación de la red aldeana". Arqueología y Territorio Medieval, 13 (1), pp. 49-94. DOI: https://doi.org/10.17561/aytm.v13i1.1519

Quirós Castillo, J. A. (2011). "Los paisajes altomedievales en el País Vasco, 500-900. De la desarticulación territorial a la emergencia de los condados". En: Quirós Castillo, J. A. (Ed.). Vasconia en la Alta Edad Media. 450-1000. Poderes y comunidades rurales en el Norte Peninsular. Documentos de Arqueología Medieval, 2. Bilbao: Universidad del País Vasco, pp. 193-218.

Reimer, P. J., Bard, E., Bayliss, A., Beck, W., Blackwell, P. G., Bronk Ramsey, C., Buck, C. E., Cheng, H., Edwards, R. L., Friedrich, M., Grootes, P. M., Guilderson, T. P., Haflidason, H., Hadjas, I., Hatté, C., Heaton, T. J., Hoffmann, D. L., Hogg, A. G., Hughen, K. A., Kaiser, F., Kromer, B., Manning, S. W., Niu, M., Reimer, R. W., Richards, D. A., Scott, E. M., Southon, J. R., Staff, R. A., Turney, C. S. M. y van der Plicht, J. (2013). "IntCal13 and Marine13 Radiocarbon Age Calibration Curves 0-50,000 Years cal BP”. Radiocarbon, 55(4), pp. 1869-1887. DOI: https://doi.org/10.2458/azu_js_rc.55.16947
Rubio Díez, R. (2020). "De Alberto del Castillo y las tumbas 'olerdolanas' a la creación de paisajes”. En: Palomino, A. y Negredo, M. (Coords.). La comunidad aldeana de Tejuela en época medieval. Arqueología funeraria y doméstica en el Alto Valle del Ebro. Burgos: Diputación de Burgos, pp. 49-65.

Sánchez-Albornoz, C. (1966). Despoblación y repoblación del Valle del Duero. Buenos Aires: Instituto de Historia de España, Universidad de Buenos Aires.

Scheuer, L. y Black, S. (2000). Developmental Juvenile Osteology. San Diego: Academic Press.

Schwartz, D. (1985). Métodos estadísticos para médicos y biólogos. Barcelona: Herder.

Séguy, I. (1997). "Aspects religieux et profanes dans le traitement funéraire réservé au nouveau-né au Moyen Âge et à l'époque moderne". En: Buchet, L. (Ed.). L'enfant, son corps, son histoire. Actes des Septièmes Journées Anthropologiques de Valbaonne. Antibes: Éditions APDCA, pp. 97-113.

Sobradillo, B., Aguirre, A., Aresti, U., Bilbao, A., Fernández-Ramos, C., Lizárraga, A., Lorenzo, H., Madariaga, L., Rica, I., Ruiz, I., Sánchez, E., Santamaría C., Serrano, J. M., Zabala, A., Zurimendi, B. y Hernández, M. (2004). Curvas y tablas de crecimiento (estudios longitudinal y transversal). Bilbao: Fundación Faustino Orbegozo.

Tente, C. (2015). “Tumbas rupestres en el Alto Mondego (Guarda, Portugal). Patrones de distribución, significados y construcción del paisaje rural altomedieval". $M u$ nibe Antropologia-Arkeologia, 66, pp. 271-290. DOI: https://doi.org/10.21630/maa.2015.66.15

Ubelaker, D. (1978). Human skeletal remains. Excavation, analysis, interpretation. Washington: Taraxacum.

Valls, A. (1985). Introducción a la Antropología. Fundamentos de la evolución y de la variabilidad biológica del hombre. Barcelona: Labor. 